\title{
Fabrication of a Novel Rotating Sample Holder to Eliminate the "Curtaining Effect" in FIB-Prepared TEM Specimens
}

\section{Nathan Wang* and George Perreault}

Maxim Integrated, 160 Rio Robles, San Jose, CA 95134

^Naiyi.wang@maximintegrated.com

\section{Introduction}

Rapidly shrinking geometries have led to a growing reliance on transmission electron microscopy (TEM) and scanning transmission electron microscopy (STEM) to image semiconductor devices because the beam spreading in thick SEM samples fundamentally limits image resolution. Focused ion beam (FIB) techniques are most often used for preparing thin TEM/STEM samples because they can easily target a particular circuit and work well across a wide variety of materials. Sample quality is the key to acquiring good STEM/TEM images. Samples need to be thin and of uniform thickness to obtain the best results.

Materials with different sputtering rates, if adjacent to the region of interest, can influence the FIB cut angle, resulting in non-uniform lamella thicknesses. This so-called curtaining effect is one of the main obstacles to obtaining high-quality images during FIB preparation of TEM samples [1]. For example, examining via/contact interfaces can be difficult if tungsten plugs are used because tungsten sputters much more slowly than the aluminum, silicon, and oxide layers adjacent to it (Figure 1).

Methods have been developed to overcome the curtaining effect $[2,3,4]$, but no technique to address this issue in less than five minutes has been published. Montoya et al. [3]

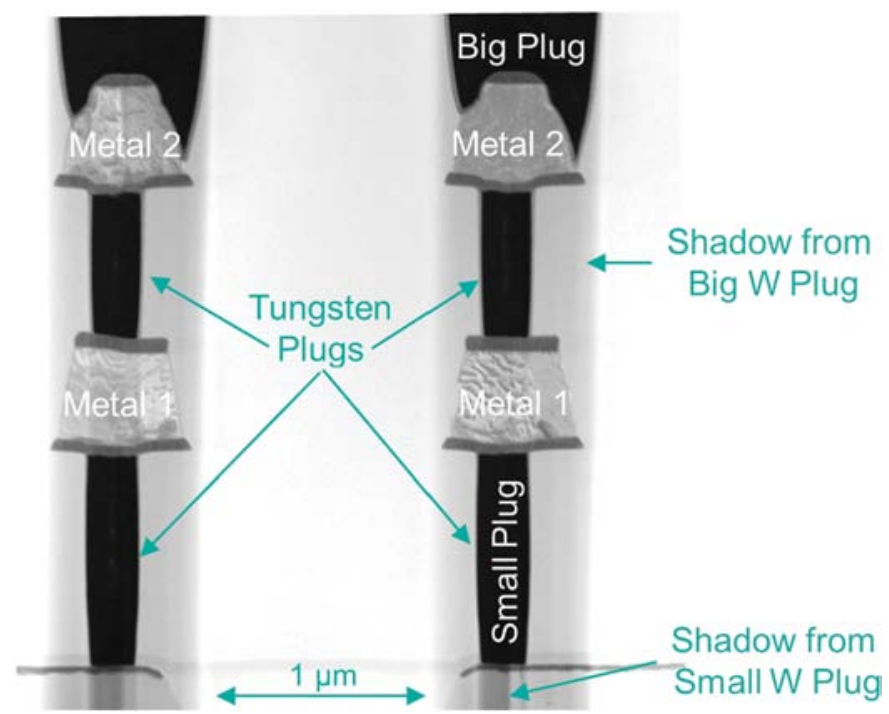

Figure 1: A STEM image showing stacked contacts, where standard FIB sample preparation resulted in the curtaining effect. The shadow area indicates sample is thicker due to the sputtering rate difference between $\mathrm{W}$ and $\mathrm{SiO}_{2}$. addressed the curtaining effect by rotating the sample to change the incident angle. This approach, though, is time-consuming and involves repeatedly mounting and removing the sample from the grid, which can result in damage to the sample. Collantes [4] proposed a sample pre-tilting method. This technique is also not optimum because most analyses require elemental identification, and this sample positioning can block X-ray access to the energy dispersive spectrometry (EDS) detector.

\section{The Challenge}

Changing the beam incident angle to the sample is an effective way to get constant thickness in the region of interest [3]. Because the FIB column is fixed, the beam incident angle can only be changed by moving the sample. In theory, the curtaining effect can be solved by flipping or rotating the sample. In practice, with existing sample movement capabilities, it is difficult to obtain the large angle changes needed and still be able to reach the region of interest with the ion beam.

A schematic sketch of the FIB system (Figure 2) is helpful in explaining why sample tilting, using the existing FIB sample movement capabilities, is not effective for solving the curtaining effect. There are two stages in the chamber, the

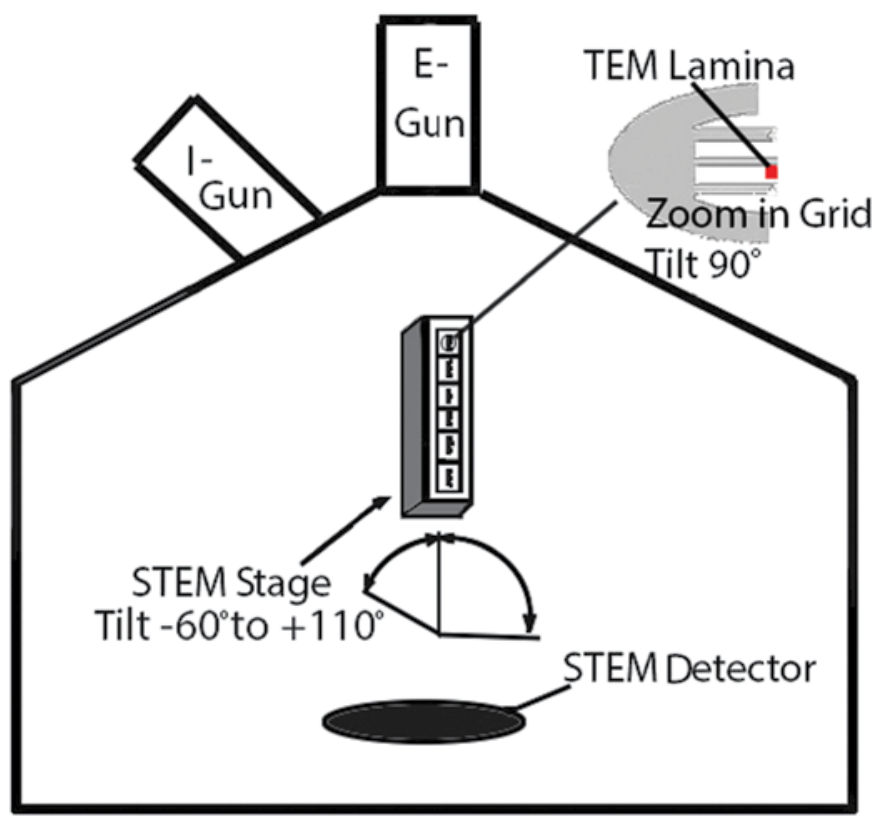

Figure 2: A FIB chamber schematic illustrating the STEM grid and sample positioning. 

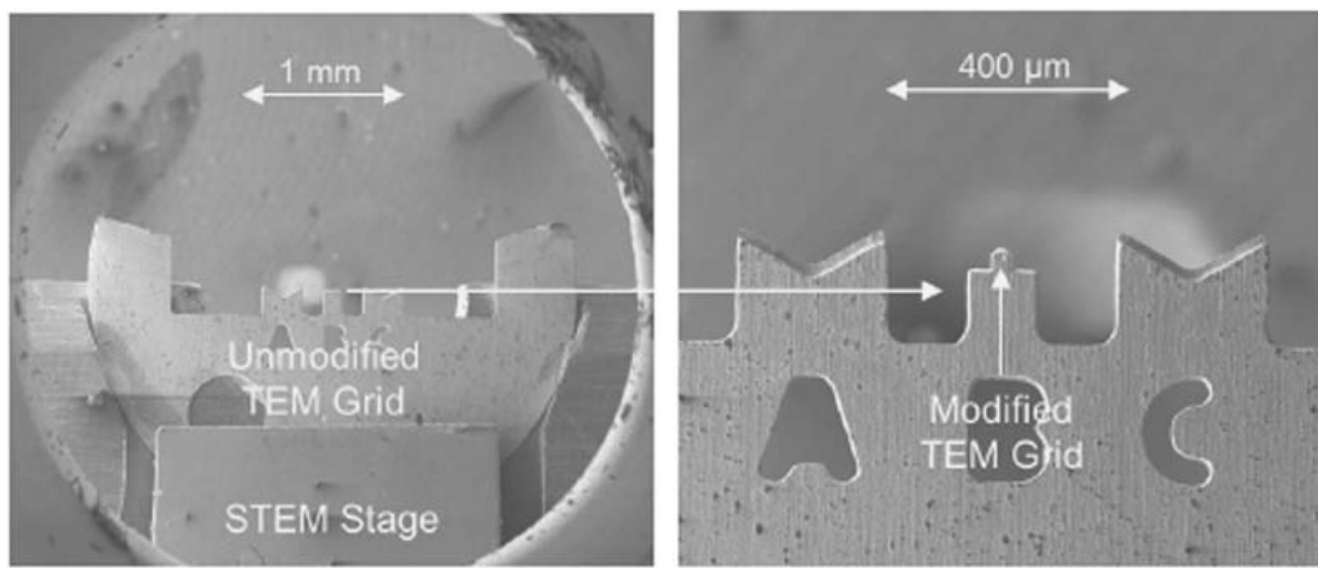

Figure 3: An unmodified (left) and modified TEM grid (right) mounted in a FIB STEM stage. Position B was modified to accommodate a new sample holder.

\section{Solution}

A "wheel" sample holder, attached to the STEM grid, was devised. The system was designed so samples can be mounted to this grid and then rotated with the use of the OMNIPROBE ${ }^{\mathrm{TM}}$. Regular machining techniques are not capable of creating such a device. A dual-beam FIB, though, is more than up to the task because it includes high-resolution viewing, precise ion-beam cutting, and gas-assisted material deposition. Micromachining in the FIB is not new: etching has been used for making logos and optical lenses [5], and the FIB's bending capability has been previously used for 3DTEM sample preparation [6].

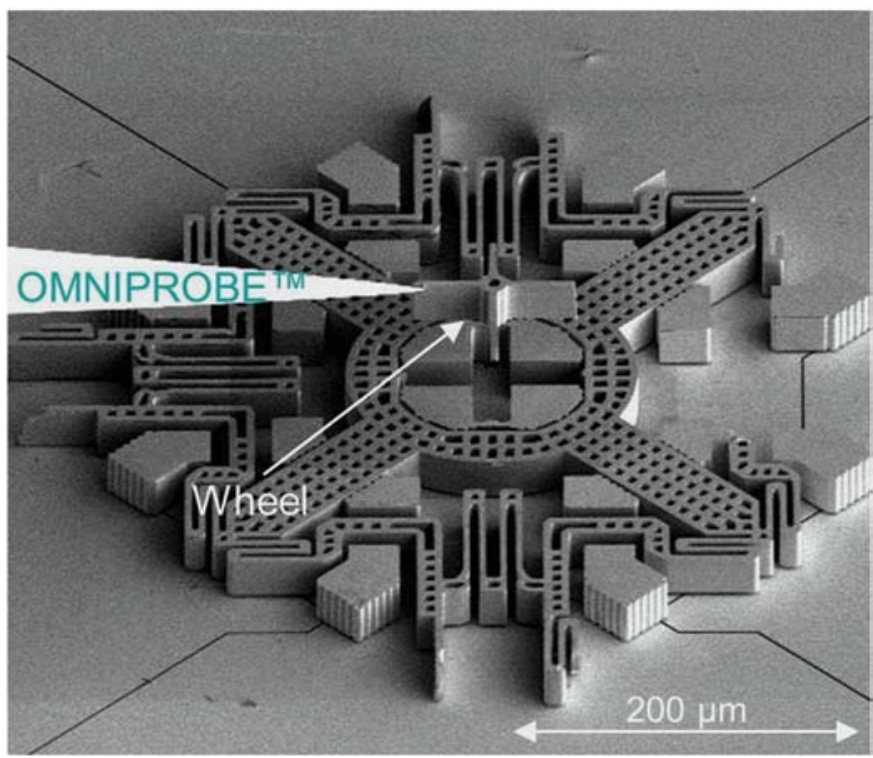

Figure 5: A wheel for the new sample holder was obtained from a MEMS device.

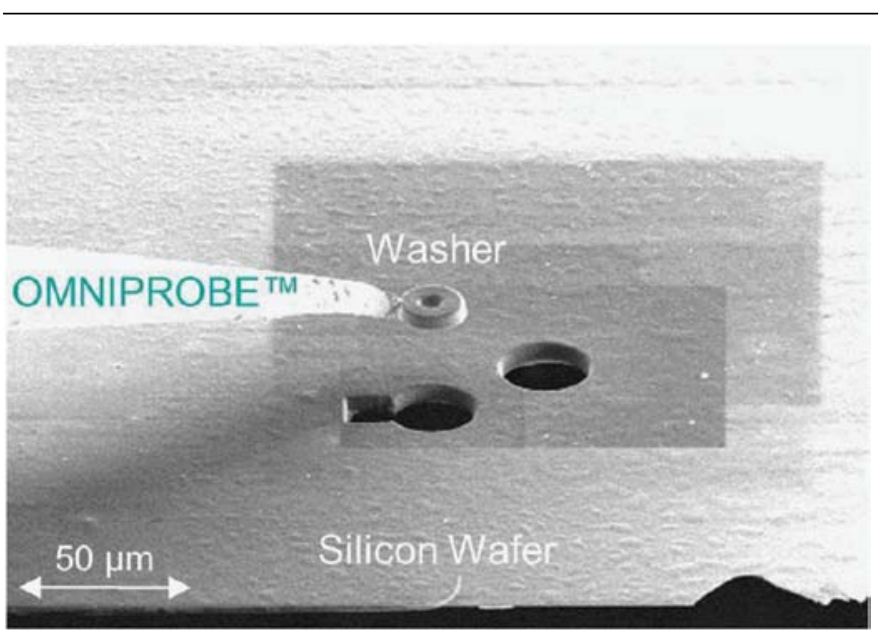

Figure 6: Annular FIB cuts through a silicon wafer, providing the fasteners used in the new sample holder.
Figure 4: A tungsten probe needle was thinned with FIB machining to provide an axle for the new sample holder.

bulk sample stage and the STEM stage. Samples are attached to an Omni STEM/TEM grid, a half-circle holder with three or five fingers for mounting lamella on the STEM stage. A half-circular grid is used to facilitate beam access to the samples. When the stage is set to $-52^{\circ}$, the ion beam is perpendicular to the sample surface, which allows ion thinning of the lamina. When the stage is set to $90^{\circ}$, the electron beam is perpendicular to the lamina, allowing STEM imaging. Tilting the sample holder is not effective for obtaining large changes to the sample-to-ion beam angle because the sample holder itself gets in the way. A sample rotation technique, which doesn't involve rotating the sample stage, is therefore needed. 


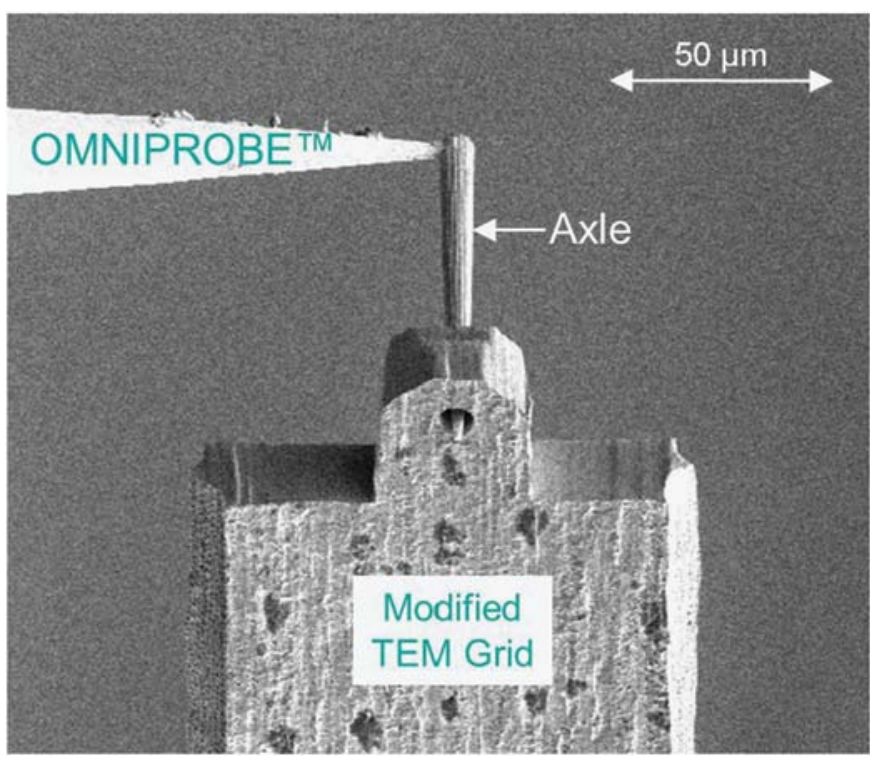

Figure 7: Assembly began by installing the axle into the modified TEM grid.

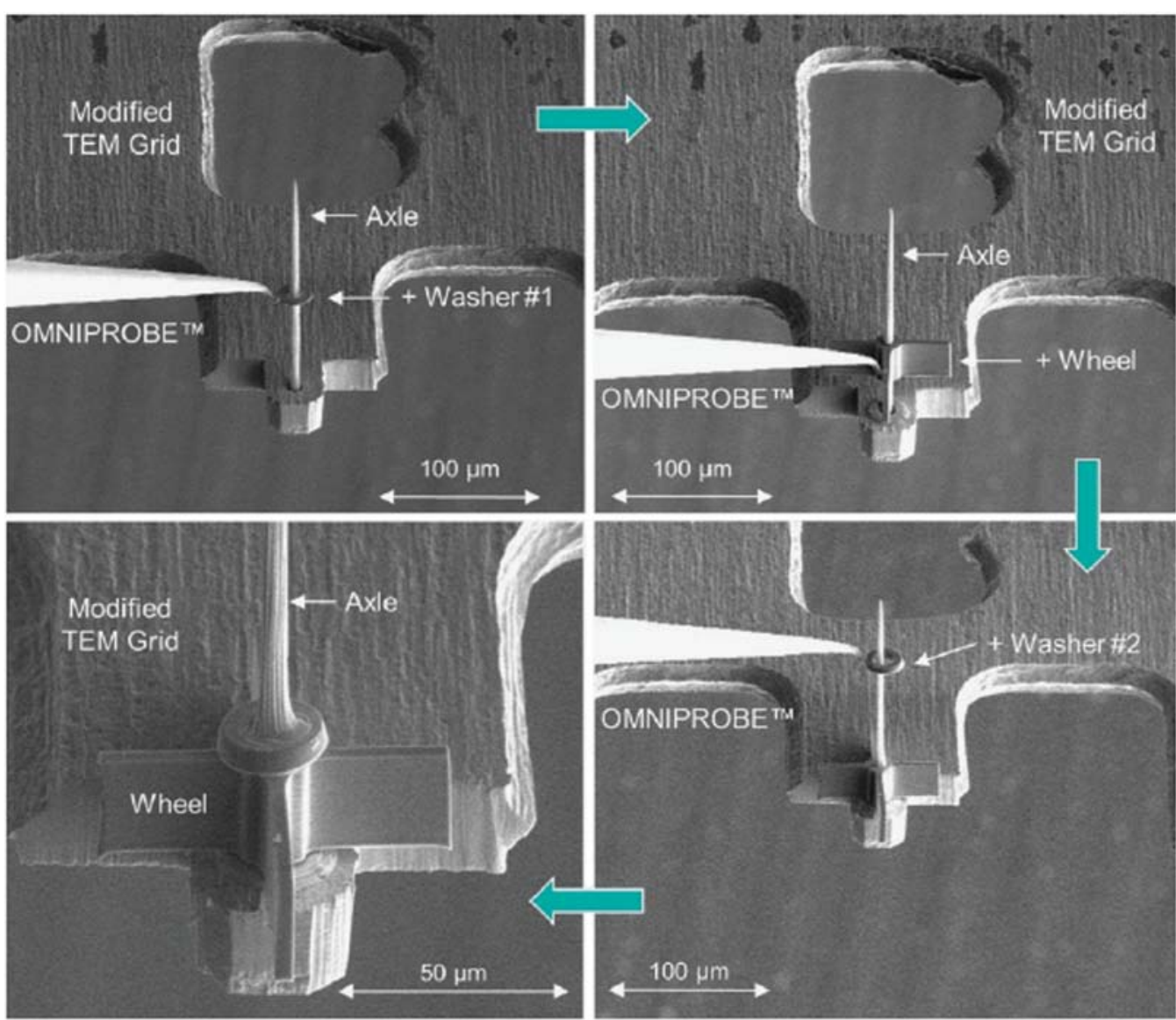

Figure 8: After axle installation, the FIB OMNIPROBE ${ }^{\mathrm{TM}}$ was used to place the fastener and the wheel. FIB-sputtered platinum was used to hold the assembly together.

A dual-beam FIB also can be used for micro-assembly. The requirements for assembling very small components includes: (1) position monitoring, (2) very precise threedimensional movement, and (3) precise attaching and detaching. Precision monitoring and precise 3D positioning are achieved by examining the object from multiple angles using the ion and electron beams for imaging. Precise cutting and depositing are easily accommodated in the FIB because these are two of its traditional functions. Positioning is well controlled during these operations because the stage and OMNIPROBE ${ }^{\mathrm{TM}}$ have submicron accuracy.

\section{Materials and Methods}

Design considerations. The basic components needed for the wheel sample holder include: a TEM grid, a wheel, an axle, and fasteners. The wheel needs to have a few long bars to attach TEM lamina. The bars should be long enough that the TEM grid will not block sample viewing.

The materials used for FIB micro-machining must be: (1) conductive, (2) stable in the FIB chamber, and (3) easy to manufacture. Conductive materials are needed to avoid charging. Some materials are not suitable for FIB use because they react with common FIB materials. Copper, for example, will be corroded by residual $\mathrm{I}_{2}$ gas, even if iodine has not been used in the FIB for 24 hours [7]. For that reason,

a molybdenum grid was selected, instead of copper, as the starting material.

Piece parts. The FIB was used to cut the molybdenum TEM grid to accommodate an axle. The center finger was reshaped, and a small hole was sputtered into its center (Figure 3). The axle was manufactured from a tungsten probe needle. Its diameter was reduced and tapered with the aid of the FIB ion beam (Figure 4). The wheel was by far the hardest component to obtain. Using FIB cuts and the OMNIPROBE ${ }^{\mathrm{TM}}$ for lift-out, a suitable wheel was scavenged from a micro-electromechanical systems (MEMS) device (Figure 5). Fasteners were obtained by machining a small washer out of a scrap piece of silicon (Figure 6).

Assembly. Basic assembly procedures involved using the OMNIPROBE $^{\mathrm{TM}}$ to pick up and place each component. Sputtered platinum was used as an adhesive. After the component was placed in the proper location, the platinum was partially sputtered away. The final bit of platinum was mechanically broken by moving the OMNIPROBE ${ }^{\mathrm{TM}}$.

Assembly starts by placing the axle in the hole of the sample grid (Figure 7). To make sure the wheel does not grind against the sample grid, a washer is added to the axle. 

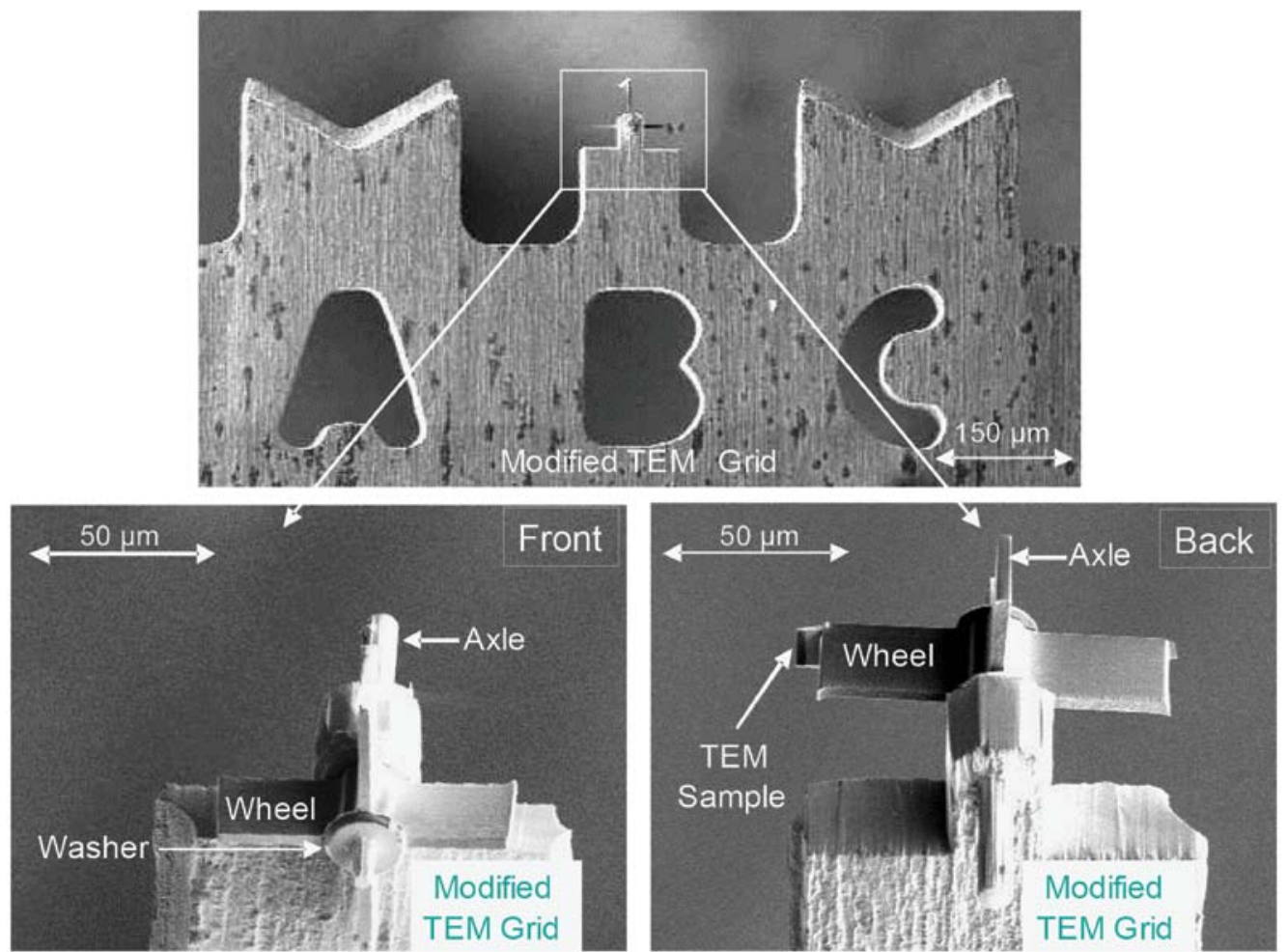

Figure 9: Front and back SEM images of the completed sample holder. Up to four samples can be mounted at the same time.
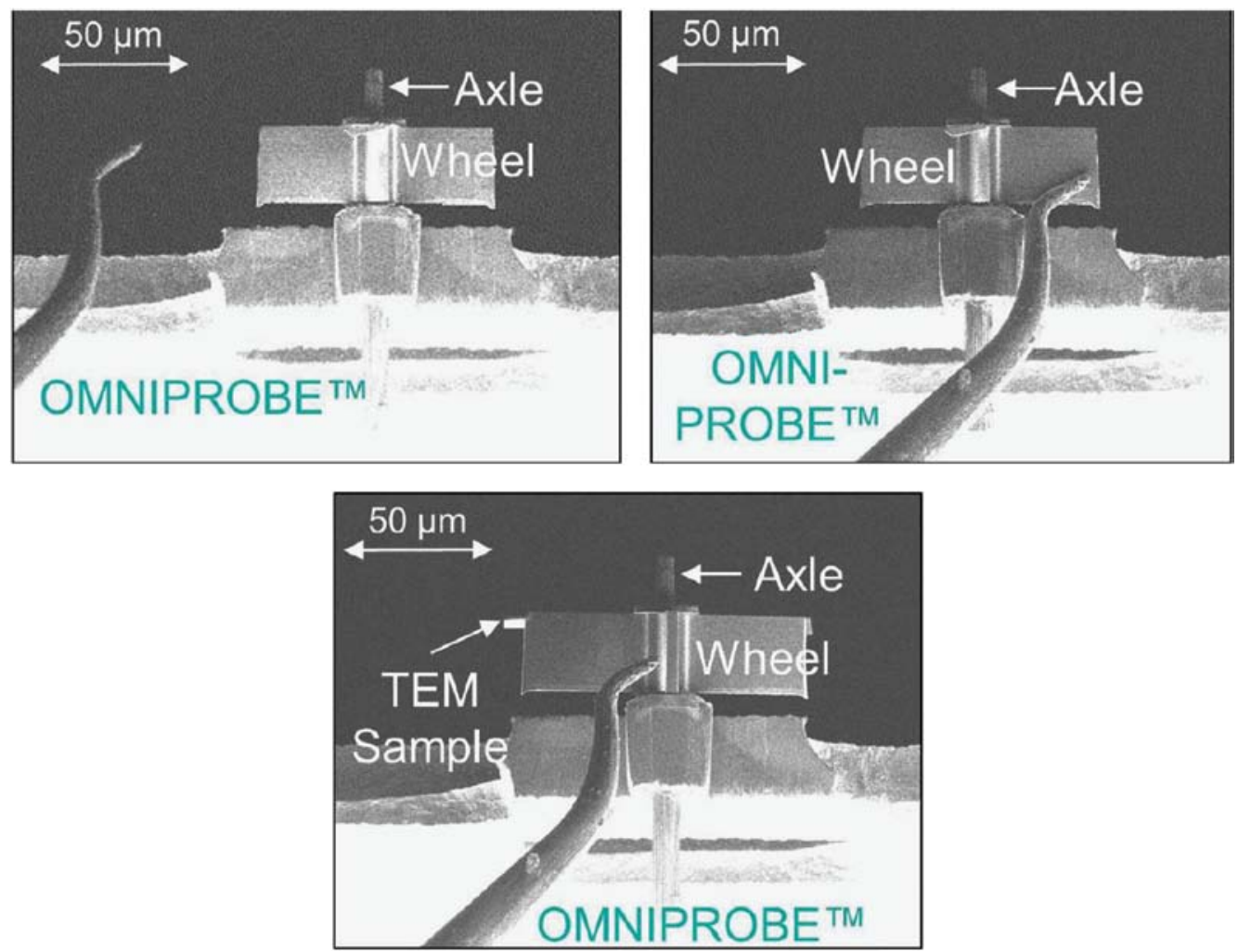

Figure 10: By pushing the wheel spokes with the OMNIPROBE ${ }^{\mathrm{TM}}$, the samples can be rotated.

Using the OMNIPROBE ${ }^{\mathrm{TM}}$ and platinum, the wheel is picked up and placed on the axle. The wheel is held in place with the silicon washer, using the same OMNIPROBE ${ }^{\mathrm{m} / \text { platinum }}$ techniques to position it into the proper location (Figure 8).

Characteristics of the sample holder wheel. Front and back images of the assembled invention are included in Figure 9. Each of the four wheel spokes can be used to hold a TEM lamina. Sample rotation is quick and easy; the OMNIPROBE ${ }^{\mathrm{TM}}$ is used to contact one of the spokes and gently turn the wheel (Figure 10). Any amount of sample rotation is possible, from $0^{\circ}-360^{\circ}$. The wheel can be repeatedly used as long as the old samples are removed. No special training is needed because any user who knows how to perform TEM lift-out is able to rotate the sample holder wheel.

\section{Results}

Tungsten plug. The stacked contacts sample image in Figure 1 was revisited after the creation of this new sample holder (Figure 11a). Within uniformly thick lamella, the region of interest under the tungsten plug was properly imaged (Figure 11b).

Wire bond failure. During the qualification of a gold-tocopper wire change, the sample wheel was used again. During this investigation, open bond wires were observed after high accelerated stress testing (HAST) $\left(130^{\circ} \mathrm{C} / 85 \%\right.$ R.H.). Conventional mechanical cross sectioning, and a follow-up SEM investigation, did not yield a root cause. Lifted bond wires were observed, but the cause was unknown.

To get to the root cause, a STEM sample was removed from the conventional SEM mechanical cross section and mounted on the rotating sample holder. Because the sample contained numerous metals (copper, aluminum, a small amount of nickel, and intermetallics), there was concern that the different sputtering rates would lead to the curtaining effect. This was avoided by using the sample wheel to align the sample during FIB thinning so the metal interfaces were parallel, and not perpendicular, to the ion beam. 

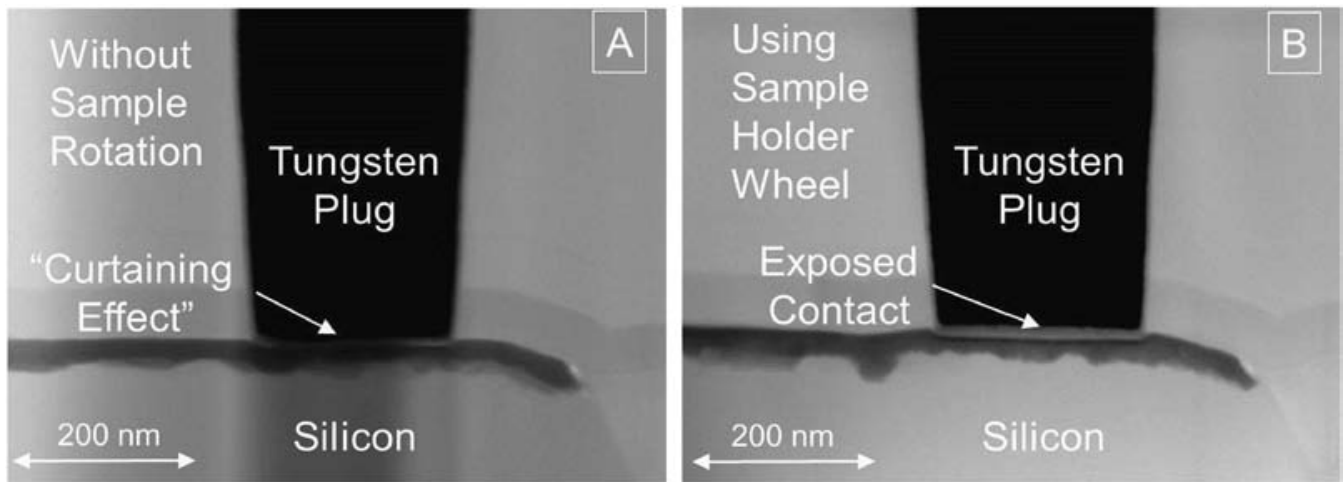

Figure 11: Stacked contacts imaged without $(A)$ and with $(B)$ the benefits of this invention. By using the Omniprobe ${ }^{T M}$ to rotate the sample $180^{\circ}$, cutting began from the silicon on the bottom of the image and not from the tungsten plug. Curtaining effects were thereby eliminated.

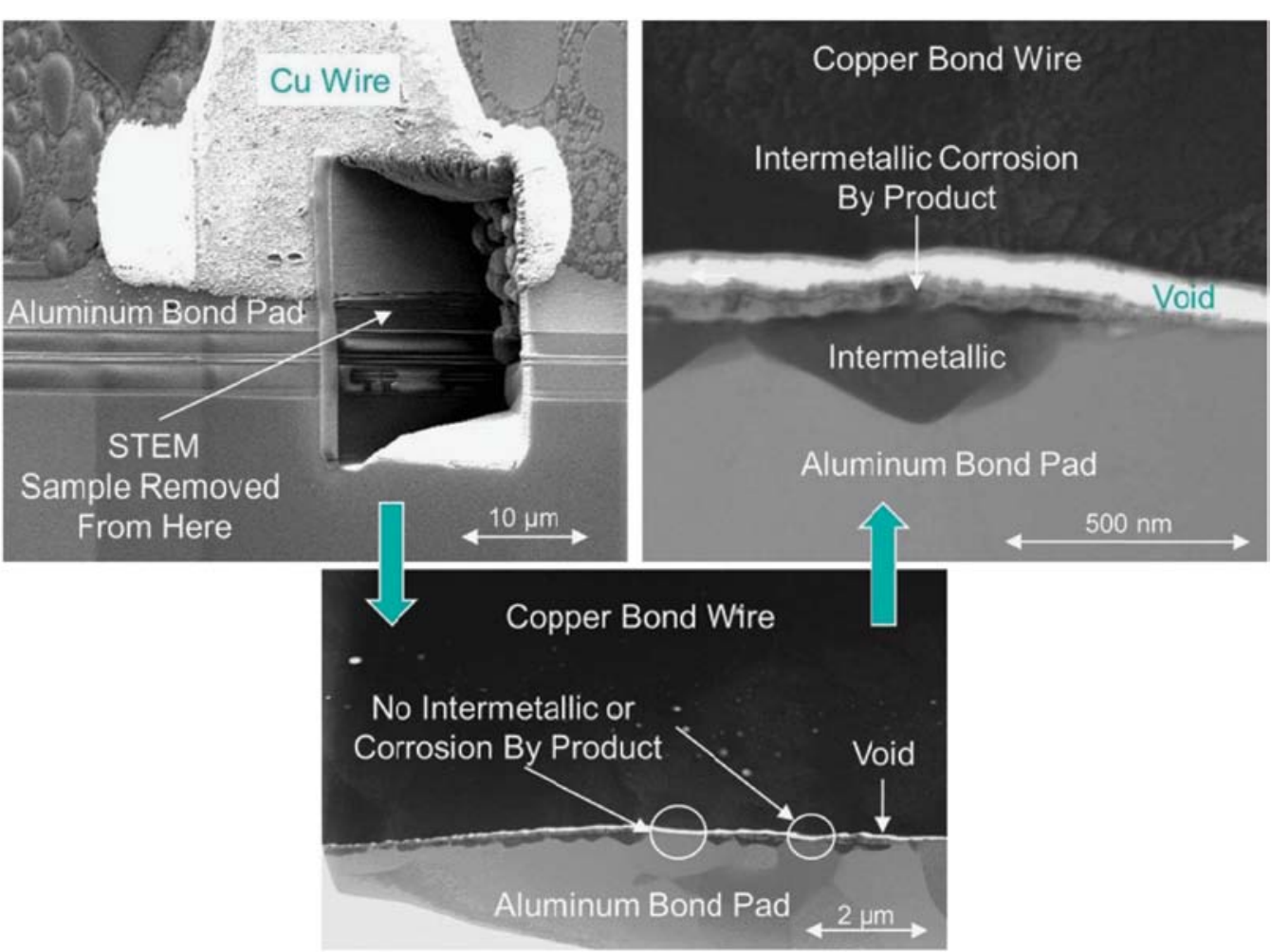

Figure 12: A STEM cross section of a copper bond wire HAST failure. Through the use of this new sample holder, both the intermetallic compound and the intermetallic compound corrosion were imaged, resulting in a complete understanding of the failure mechanism.

Wire bond lifting and intermetallic compound formation were observed in the STEM sample (Figure 12). Because there was some voiding, the intermetallic compounds were not observed everywhere. A second thinner $(\sim 50 \mathrm{~nm})$ layer was observed on top of the much thicker intermetallic layer. Most tellingly, this unknown layer was observed only on the intermetallic and was not seen over the aluminum bond pad, strongly implying that the cause was not simple contamination prior to wire bonding since all surfaces were not affected. From EDS, it was observed that both the intermetallic and unknown layer had similar composition, except the unknown layer was richer in oxygen. It was subsequently concluded that this $\sim 50 \mathrm{~nm}$ layer was a corrosion product of the intermetallic compounds caused by the high heat and humidity of the HAST stress test. Without a properly prepared sample, made possible by the rotating sample wheel, this important conclusion would not have been reached.

\section{Conclusion}

A new sample holder to solve the curtaining effectassociated with FIB preparation of TEM samples was presented, and construction details were described. Because the sample holder was assembled from items in the lab and the OMNIPROBE $^{\mathrm{TM}}$ is a standard attachment for dual-beam FIBs, the only significant cost is the operator's time. The tool has been shown to be both effective and easy to use.

\section{References}

[1] U Muehle et al., "FIB-Based Target Preparations of Complex Material Systems for Advanced TEM Investigations". in Microscopy: Science, Technology, Applications and Education, eds. A Méndez-Vilas, J Díaz, Formatex Research Center, Badajoz, Spain, 2010.

[2] CA Volkert et al., MRS Bull 32 (2007) 389-99.

[3] E Montoya et al., Microsc Res Techniq 70 (2007) 1060-71.

[4] Angela Collantes, "A New Method to Reduce Artifacts During TEM Sample Preparation Using FIB," European FIB User Group Meeting 2006, Wuppertal, Gemany, Oct. 2006.

[5] J Puretz et al., Focused Ion Beam Micromachining of Optical Surfaces in Materials. US Patent 4,698,129, filed May 1, 1986, issued Oct. 6, 1987.

[6] N Wang, Microscopy Today 13 (2005) 36-39.

[7] $\mathrm{H}$ Bender et al., "Investigation on the Corrosion of $\mathrm{Cu}$ Metallization in the Focused Ion Beam System Due to a low I2 Background", ISTFA 1999: Proceedings of the 25th International Symposium for Testing and Failure Analysis (ASM International), (Oct. 1, 1999) $135-40$. 